\title{
On the Nature of Space and the Emergence of the New Physics Some Speculations
}

\author{
Philip J. Tattersall ${ }^{1}$ \\ ${ }^{1}$ Private researcher, 8 Lenborough St, Beauty Point, Tasmania, Australia \\ Correspondence: Philip J. Tattersall, 8 Lenborough St, Beauty Point, Tasmania, Australia. E-mail: \\ soiltechresearch@bigpond.com
}

Received: September 26, 2016

Accepted: October 18, 2016 Online Published: November 17, 2016

doi:10.5539/apr.v8n6p25

URL: http://dx.doi.org/10.5539/apr.v8n6p25

\begin{abstract}
In reflecting on the emergent ideas of Stochastic Electrodynamics (SED) the author suggests that the zero point activity, also termed 'vacuum noise,' is not only a fundamental aspect of space, but is space itself.

Recent experiments are discussed in which electromagnetic radiation has been shown to cause changes in gravitational effects. This essay speculates that the zero point energy may be responsible.
\end{abstract}

Keywords: space, virtual activity, stochastic electrodynamics, Zero Point, ZPE, ZPF, quantum, gravity, vacuum

\section{Introduction}

For many years there has been ongoing questioning as to the 'real' nature of space (Johnson \& Walker, 2005). For its part classical physics assumes space is a vacuum; nothingness; a void. Quantum theory on the other hand posit that space, by necessity is full of 'virtual activity', with elementary particles being created and annihilated instantaneously, resulting in an overall void. By admitting such activity, even though virtual, the door was opened to ongoing questioning, experimental work and attendant theorizing. The subsequent schools of thought, fueled by the results of experimental work, have now taken us to the point where questions over the nature of space are, more than ever, ripe for consideration.

Increasingly researchers have embraced the idea that far from being merely a state of virtual activity space is actually full of real activity or quantum 'noise' (Davies, 2001). Researchers have termed the activity Zero Point Field (ZPF), meaning the complex and random radiation field, made up of zero point energy (ZPE) still present at absolute zero (de La Pena et al., 2012). Boyer (2015, pp. 13-14) provides a useful definition, “...zero-point radiation and thermal radiation are treated on the same footing in classical electromagnetism, they have different effects because of the differences in the electromagnetic spectra at zero temperature and finite temperature. The spectrum of zero-point radiation holding at zero temperature is Lorentz invariant, scale invariant and invariant under adiabatic compression. The zero-point radiation spectrum is isotropic in any inertial frame. In free space, the zero-point spectrum cannot give rise to velocity-dependent forces on particles because of its Lorentz-invariant character...in contrast, the spectrum of thermal radiation at a non-zero temperature...has a preferred reference frame, that unique frame in which the spectrum is isotropic. At finite non-zero temperature in free space, the thermal spectrum gives velocity-dependent forces on any system having electromagnetic interactions which is moving through the thermal radiation."

de la Pena et al. (2012, p.1) regard the ZPF as largely responsible for quantum phenomena and posit that inclusion of the ZPF in classical physics would lead to the emergence of quantum theory, thus securing the vital link between the macro and micro 'worlds'. They claim, "In other words, that rather than being innate, the quantum phenomenon arises as a consequence of the interaction of the zero-point field with matter. The recognition of the reality of this field that fills the whole of space even at null absolute temperature, not only allows to better understand (rather than merely describe) the quantum phenomenon, but also opens the door to some new physics".

Although the idea is a relative newcomer to modern physics the theoretical underpinnings have been around since the 1960's when Marshall (cited in de la Pena et al., 2012) and Boyer (cited in de la Pena et al., 2012) developed the theoretical basis of Stochastic Electrodynamics (SED) (see Note 1). The key assumption germane to this essay is that SED takes the existence of the ZPF as real, seeing it as a classical background radiation field 
consistent with Maxwell's equations, with stochastic amplitude and it is assumed that its stochastic properties are the same for every inertial frame of reference (Hernandez, 2014).

In terms of 'wave-particle' phenomena, the SED theory maintains inter alia that an interaction between particles in motion and the ZPF causes the emergence of an electromagnetic wave that 'guides' particle trajectory. The apparent particle-wave duality and entanglement are thus explained. Marshall and Santos (1997) further elaborated on the role of the ZPF in the context of a wave description of light. They show how light waves and the ZPF interact under certain experimental conditions to yield correlated amplification outputs. In an analysis of the anticorrelation beam splitter experiments of Grangier, Roger and Aspect (1986), Marshall and Santos (1997, p.5) state, “...It is easy to see, qualitatively, how the explanatory power of a purely wave theory is increased by the recognition of a real zero point field. A beam splitter does not simply split an incoming wave into parts; it mixes together two incoming waves, one of them from the 'vacuum', to give two outgoing waves...” (see Note 2). (Bolded text is the original the author's emphasis).

de la Pena and Cetto (1996) have also elaborated the ZPF theory at some length over many years. Likewise Santos (2012, p.5), in keeping with his extensive and important contributions to the field, has recently presented cogent arguments regarding the role and nature of ZPF. In discussing the SED nature of the ZPF he states that fluctuation inherent in the ZPF, “...gives rise to two characteristic traits of quantum physics, Firstly quantum theory should be probabilistic. Secondly it presents a kind of wholeness, quite strange to classical physics where the concept of isolated system is crucial. The fact that the vacuum fluctuations at different points maybe correlated is the origin of wholeness. Indeed entanglement may be a consequence of the correlation of quantum fluctuations at different points", (bolded text is the original author's emphasis).

The idea of wholeness opens the door to considerations of possible ZPF interactions with matter. Santos (2012) posits that atoms are in dynamic stationary state with the fluctuating ZPF, citing the electronic stability of the hydrogen atom (and atoms generally) as an example where the electron is essentially in a kind of resonance with an energy level within the ZPF. Boyer (2011), citing the theoretical calculations of other workers has elaborated on the idea that ZPF is responsible for electronic stability. Calculations on probability distribution have shown agreement (for hydrogen) with Schrödinger calculations.

These two key points relating to wholeness and dynamic energy source are thought to be fertile ground.

\section{The ZPF and the Nature of Space}

These speculations (along with the theoretical underpinnings of SED) would lead one to suggest that space is not full of ZPF, but rather that space is in fact ZPF. This is not a return to the aether theory of old, but is an acknowledgement of a mounting body of theoretical argument and experimental work suggesting that 'space' is in fact an active, all pervasive medium.

If we assume that space is full of activity and that the ZPF does interact with particles, fields and matter then it would seem plausible to extend its influence beyond the micro (quantum) to the macro world. In building on the ideas of Santos (2012), that mass is interacting with the ZPF it could be suggested that the effect of gravity may emerge from such interactions. Indeed there has been a considerable amount of research, discussion and theorizing regarding the ways in which ZPF energies (ZPE) interact with matter. Dirac (1951) concluded (rather provocatively), "Thus with the new electrodynamics we are rather forced to have an aether". Dirac makes an interesting point and it has resonated with a number of researchers including Haisch, Rueda \& Puthoff (1997). Haisch, Rueda and Puthoff immediately saw the ZPF as an electromagnetic component of Dirac's aether, stating that "Ether is a loaded term today because it is so widely described as an outmoded, discredited idea. The $19^{\text {th }}$-century ether was an ill-defined 'substance' providing a universal frame of rest and a medium for propagation of light waves. The ZPF is not an ether of this sort. The ZPF is light waves... . Being Lorentz invariant it cannot and does not act as a universal frame of reference for rectilinear motion...However it does provide a universal frame of rest vis-a-vis acceleration..." (Haisch, Rueda \& Puthoff, 1997, p.8). In their recent work, Rueda and Haisch (2005) have shown how the electromagnetic quantum vacuum field (or ZPF) makes a contribution to inertial reaction force of an accelerated object. The authors present an elaborate set of theoretical arguments in support of their Quantum Vacuum Inertia Hypothesis, in which they have put forward detailed calculations and discussion relating to the connection of inertia and gravity to the vacuum fields.

This as it turns out is a most useful starting point in explaining the results of recent experiments involving the effects of gravity on selected masses. 


\section{A Speculation on the Cause of Gravity}

While general relativity (GR) produces accurate predictions it remains silent as to the cause of gravity. Over many years there have been numerous attempts to elucidate the cause of gravity. For example, the recent efforts by Verlinde (2010) although seen as somewhat controversial, have sparked considerable interest and research activity. Verlinde posits that gravity emerges as an entropic force brought about by changes in the information associated with the position of bodies. While aspects of Verlinde's arguments strike chords with the key speculations in this paper, one senses that the cause still eludes us. The danger is that many of the candidate theories may effectively be surrogates for a deeper theory, in that they can make accurate predictions and are often in agreement with GR, but lack a clear explanation as to the cause of gravity.

Rueda and Haisch (2005) in drawing on the theoretical arguments of Sakharov and others pointed to the idea that gravity emerges from the vacuum, and in fact may arise from the effect of massive bodies on the permeability and permittivity of space itself (Puthoff, 2001). This view is close to the one I am suggesting, namely that space is ZPF; in a sense a real physical presence that due to its characteristics remains of itself undetectable, but whose effects emerge in the presence of matter.

Let us speculate that certain ZPE modes within the ZPF are continually moving into matter in order to provide an energy source for electron activity and possibly other activity within atoms. It would follow that the intensity of movement of the ZPE would be proportional to both mass and distance. The net result would be a distortion of space in the vicinity of massive bodies. On the face of it the mathematical approach used in GR would still apply.

The gravitational effect resulting from the ZPE-mass interaction would produce a slight thermal effect leading to an entropic emission, perhaps contributing to dark energy. The thermal effect is a significant thermodynamic consideration. As pointed out by Van Flandern (1996) the absorption of 'particle flux' by matter would result in heating effects. He noted that, the six largest planets all appear to radiate more heat back into space than they take from the sun. It has been traditional to attribute this excess to radioactivity in the planetary cores, although no observation evidence supports this conjecture...".

In a very real sense gravity would emerge from space and would appear to do so in the presence of matter. Therefore gravity is not a property of mass or space as such, but rather emerges as a consequence of the mutual interaction of ZPE modes within the ZPF (space) and matter.

\section{An Interesting Experimental Development}

The findings by Rancourt (2011), arising from experiments he conducted over a period of many years (in which he experimented with torsion pendulums and masses) suggest that light somehow interferes with the effect of gravity. Placing a laser light in the form of a curtain of light to one side of a mass on a torsion pendulum he was able to show that the mass moved toward the light. The elegant experiments, confirmed many times under a range of conditions led to the suggestion that gravity is in fact a pushing force (Rancourt, 2014). The movement of the test mass towards the light suggested that either the light was attracting the mass or that some kind of external 'influence' was blocked by the light thus creating a disequilibrium leading to movement of the mass, caused by an influence from the opposite (non-light) side. Professor Rancourt eliminated the effect of light as an attractor by housing it in a steel box. The effect was the same even though a number of different test mass materials were used.

If the movement of test masses is due to a pushing force then it is possible, based on my earlier suggestion, that the ZPF has been somehow affected by the light in the experiment (e.g. perhaps through correlative effects such as those discussed earlier in this paper (Marshall \& Santos, 1997)) leading to a disequilibrium and the emergence of pushing forces. The pushing force hypothesis was further explored in experiments reported by Rancourt and Tattersall (2015). The authors speculated that zero point energy within the zero point field may be responsible for so called gravitational 'attraction'. As mentioned above, the movement of ZPE into matter may well be the cause of 'gravity' as bodies in proximity to each other are actually driven together by the pressure of space itself due to the movement of ZPE into matter in order to maintain atomic processes. Such a hypothesis would be contingent on stringent thermodynamic considerations.

\section{Conclusions}

This brief essay attempts to put forward a series of ideas in the form of speculations based on past and present developments in SED. Recent interesting experiments may prove to break new ground in our understanding of the nature of space as an active energetic influence that in the presence of matter leads to the emergence of gravity and inertia. The influence (zero-point energy) has been found to be Lorentz invariant, scale invariant and 
invariant under adiabatic compression and active at zero Kelvin (absolute zero). The emergence of gravity and inertial resistance are caused by the interaction of ZPE with leptons and possibly quarks at the atomic level (Haisch et al., 1997), thus the micro world of the quantum is linked to the macro world in a new concept of wholeness.

A quote from Santos $(2012$, p.1) is food for thought as we continue on jour journey toward the new physics, "Understanding quantum mechanics presents big difficulties for many people, a paradoxical fact in view of the relevance and practical success of the theory. For physicists interested in applications understanding is not very relevant if it means getting an intuitive picture of the micro-world. In fact, for them the essential purpose of physics is to allow predicting the results of experiments. Other physicists, including most workers in foundations, think the real problem is that, in the attempt to understand quantum mechanics, we should not use concepts of classical physics, or we should adhere to a kind of 'weak' objectivity. In contrast with those opinions,...it is proposed that quantum mechanics is less different from classical physics than usually assumed, and might be understood in a similar manner." To my mind the answers to many of the fascinating, and some say troubling questions facing physics may be closer than we think and may involve seeing what we have already seen in new ways.

It is without doubt that the growing field of SED is providing exciting and much needed opportunities for canvassing new ideas, and indeed old ideas anew!

By encouraging the publication of essays such as this the scientific community displays a willingness to entertain new ideas and intuitive speculations that may well help point toward a new way for physics. The joy is not so much in knowing, but is in the journey to the knowing.

\section{References}

Boyer, T. (2011). Any classical description of nature requires classical electromagnetic zero-point radiation. Retrieved from https://arxiv.org/abs/1107.3455v1

Boyer, T. (2015). Understanding zero-point energy in the context of classical electromagnetism. Retrieved from http://arxiv.org/pdf/1512.08033v1.pdf

Davies, P. C. W. (2001). Quantum vacuum noise in physics and cosmology. Chaos, 11(3). http://dx.doi.org/ $10.163 / 1.1378796$

de la Pena, L., \& Cetto, A. M. (1996). The quantum dice. An introduction to stochastic electrodynamics. Dordrecht, The Netherlands: Kluwer Academic Publishers.

de la Pena, L., Cetto, A. M., \& Hernandez, A. V. (2012). Whence the quantum? Retrieved from http://fqxi.org/community/forum/topic/1384

Dirac, P. A. M. (1951). Is there an aether? Nature, 168.

Grangier, P., Roger, G., \& Aspect, A. (1986). Experimental evidence for a photon anticorrelation effect on a beam splitter: a new light on single-photon interference. Europhysics Letters, 1(4).

Haisch, B., Rueda, A., \& Puthoff, H. E. (1997). Physics of the zero point field: implications for inertia, gravitation and mass. Speculations in Science and Technology, 20.

Hernandez, C. A. D. (2014). Wave duality from stochastic electrodynamics. Retrieved from https://arxiv.org/pdf/ 1403.0016v1.pdf

Johnson, G. W., \& Walker, M. E. (2005). Sir Michael Atiyah's Einstein lecture: “The Nature of Space”. Notices of the AMS, 53(6).

Marshall, T., \& Santos, E. (1997). The myth of the photon. Retrieved from http://arxiv.org/pdf/quant-ph/971 1046v1.pdf

Puthoff, H. E. (2001). Polarizable-vacuum (PV) representation of General Relativity. Retrieved from http://lanl.arxiv.org/ftp/gr-qc/papers/9909/9909037.pdf

Rancourt, L. (2011). Effect of light on gravitational attraction. Physics Essays, 24(4). http://dx.doi.org/10.4006/ 1.3653936

Rancourt, L. (2014). Why I think gravity is a pushing force. Gravityforces. Retrieved from http://www.gravityforces.com/?author=1

Rancourt, L., \& Tattersall, P. J. (2015). Further experiments demonstrating the effect of light on gravitation. Applied Physics Research, 7(4), 4-13. http://dx.doi.org/10.5539/apr.v7n4p4 
Rueda, A., \& Haisch, B. (2005). Gravity and the quantum vacuum inertia hypothesis, Ann. Phys. (Leipzig), 14(8). http://dx.doi.org/10.1002andp.200510147

Santos, E. (2012). Vacuum fluctuations, the clue for a realistic interpretation of quantum mechanics. Retrieved from https://arxiv.org/pdf/1208.4431.pdf

Van Flandern, T. (1996). Possible new properties of gravity. Meta Research Bulletin, 5(38). (Note 3)

Verlinde, E. (2010). On the origin of gravity and the laws of Newton. Retrieved from https://arxiv.org/abs/1001. 0785

\section{Notes}

Note 1. Boyer (2015) provides a detailed and useful discussion of the zero point energy that is accessible and presents cogent mathematical arguments

Note 2. Marshall and Santos (1997, p.5) summarize the findings of the Grangier, Roger and Aspect beam splitter experiments in terms of the ZPF as, "If we consider the 'vacuum' as empty then it seems almost unavoidable to assume that the intensity of any incoming classical signal is equal to the sum of the intensities in the outgoing channels... with such a description it is not possible to explain the anticorrelation data; these were interpreted by Grangier, Roger and Aspect as evidence that the whole 'photon' goes onto one or the other of the outgoing channels."

Note 3. Reprinted from Astrophys. \& Space Sci. 244. (1996): http://dx.doi.org/10.1007/BF00642296

\section{Copyrights}

Copyright for this article is retained by the author(s), with first publication rights granted to the journal.

This is an open-access article distributed under the terms and conditions of the Creative Commons Attribution license (http://creativecommons.org/licenses/by/4.0/). 